\title{
ScIDoC
}

\author{
International Journal of Dentistry and Oral Science (IJDOS) \\ ISSN: 2377-8075
}

\section{Management of Compromised Esthetic Zone with Modified Bio-Col Procedure}

Research Article

Rajeev Arunachalam*, Vini Rajeev, Sivadas Ganapathy, Vaishnavi Vedam

Senior Lecturer, Faculty of Dentistry, Asian Institute of Medicine, Science and Technology (AIMST) University, Malaysia.

\section{Abstract}

Following extraction of tooth, a considerable change in alveolar ridge anatomy occurs pertaining to morphology and dimension in a short span of time. These variations can significantly affect future rehabilitation process if not planned. An implant placement may become more complicated if bone resorption is more. Tissue engineering aims to reestablish functional, healthy tissues and structures in order to supplant unhealthy, dying, or deceased tissues. Diverse tissue regenerative techniques have been established for rectifying bone defects with the intention of creating an efficient and appealingly agreeable pontic or implant restoration situates. A simple minimally invasive procedure immediately following tooth extraction known as Bio-Col alveolar ridge preservation technique decreases the necessity for reestablishing challenging ridge defects. This case represents a revised technique of preserving socket in periodontally compromised situation using a combination of platelet rich fibrin, collagen scaffold and bone graft followed by rehabilitation with implant for esthetic replacement. Clinically and radiographically efficacious restoration of the alveolar ridge, with the ability to receive functionally and esthetically acceptable implant site was demonstrated.The modification simplifies the technique without negotiating the esthetic outcome. This procedure is beneficial in maintaining alveolar bone and soft tissue architecture.

Keywords: Atraumatic Extraction; Esthetics; Fixed Partial Denture; Implant; Osseointegration.

\section{Introduction}

Periodontally compromised tooth with hopeless prognosis may institute therapeutic extractions. The functional and esthetic replacement of the teeth being removed and their supporting tissues should be taken into prior notice, when extractions are planned. Fixed bridge options were the benchmark for esthetic replacements previously. Increased awareness and widespread use of dental implants have made preservation of both alveolar bone and essential gingival contours possible for esthetic restoration. Use of minimally invasive technique ensures an atraumatic extraction which is the first possible step ensuring a better esthetics and function. This holds true if an implant is planned for extraction site. Adopting an appropriate technique enables the treatment in a shorter time and with fewer surgical procedures thereby preserving the alveolar ridge anatomy, aiding the prosthetic management. Ridge preservation should be considered in strategically essential sites to maintain the prospect of an implant preference for the patient in the future.

The goal of the site-preservation technique is to minimize damage of alveolar ridge contours secondary to osseous remodeling, increase bone regeneration within the extraction socket, and prevent soft tissue collapse associated with tooth loss. The bio-col alveolar ridge preservation procedure takes the benefit of the synergistic effect of merging surgical and prosthetic site- conservation protocols. The surgical procedure makes sure the conservancy of both hard and soft tissues at the period of tooth removal, and it reduces or eradicates the bone resorption that normally follows. The prosthetic protocol uses interim restorations to support the soft tissues neighboring the extraction site, thereby averting the ridge collapse during maturation. The usage of grafting material in any stoichiometric form is suggested as it clues to reduced resorption of alveolar crest. The advantage using an array of regenerative materials like bone graft within a collagen scaffold will give more structured bone with no signs of in growth other

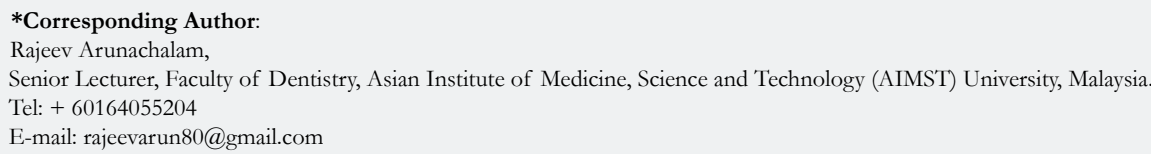

Copyright: Rajeev Arunachalam ${ }^{2021}$. This is an open-access article distributed under the terms of the Creative Commons Attribution License, which permits unrestricted use, distribution and reproduction in any medium, provided the original author and source are credited. 
than bone. This may be related to collagen material functioning as a barrier to the ingrowth of surrounding tissue that could have impeded the process of bone regeneration. The original Bio-col technique grosses the benefit of the above principle and recommends atraumatic extraction without perforation of the socket wall followed by condensation graft and collagen material [1].

The natural healing phenomenon after tooth removal involves resorption and remodeling of the alveolar ridge, which is physiologically detrimental plus certainly inevitable and can have negative impact on implant placement $[2,3]$. This holds true in maxillary anterior region, where the vestibular wall can be damaged more frequently during tooth removal due to prominent root position which is generally accompanied by an extremely fine and fragile buccal wall [4]. The maturation and mineralization of the recently formed bone in the extraction socket can be hastened or enhanced by ridge preservation. In areas where there is an osseous defect, the predictability and outcome of the implant procedure is largely determined by the shape of the defect. Pertaining to extraction sites the osseous defects can be classified based on morphology as favorable or unfavorable [5]. There is distinct treatment protocol variability among above mentioned defects. In favorable sites additional site preparation surgeries are not required during implant placement whereas the unfavorable defects at aesthetic sites mandates site- development procedures.

Creating a strong foundation for osseointegrated implants by preserving the socket and alveolar ridge anatomy is the primary purpose of surgical bone grafting techniques [6]. It is important for dentist to decide at what time and where forthcoming implants must be located. For the above an understanding of hard and soft tissue alterations of post-extraction sockets and the form of anatomy of the future implant site is needed. Not only osseointegration but also an appealing restoration that is unwavering over time is the current focus for implant therapy [7]. Preoperative planning of various factors should be made and the scheduling of implant placement post-extraction is crucial which influences the aesthetic outcome [8].

This clinical report highlights the rehabilitation of maxillary anterior tooth of a young male patient with a history of tooth loss due to road traffic accident in a periodontally compromised tooth using modified bio-col technique and subsequent prosthodontic management with dental implant.

\section{Case Presentation}

A 26 year old male patient was referred to prosthodontist with a complaint of mobility of upper left central incisor following a road traffic accident. Dental history was significant for recent trauma to upper left central incisor in a road traffic accident but the tooth had not been avulsed. There was no significant medical history. Upon assessment and diagnosis of the traumatized tooth, it was determined that the tooth showed grade II mobility with supraeruption. Patient's history revealed that prior to accident the spacing was seen been two upper central incisors and was gradually increasing with mild supraeruption in relation to upper left central incisor. Radiographic assessment was done and angular pattern of bone loss was seen in relation to distal aspect of root extending more than half of root length (Figure 1). Since the patient was seen on an emergency basis the traumatized tooth had been splinted to the adjacent teeth using composite resin and wire (Figure 1). The patient was subjected to complete scaling and thorough root planning under LA and oral hygiene instructions were given and was decided to be reviewed after 4 weeks to evaluate the prognosis and definite management. Following 4 weeks, evaluation was done and as the grade of mobility and supraeruption worsened, the tooth was deemed hopeless and planned for extraction. Restoration options included endosseous implant in relation to upper left central incisor, fixed partial denture, or removable partial denture (RPD). The patient voiced a strong need to have the tooth replaced with implants. The patient was nonsmoker.

As the tooth had periodontal involvement atraumatic extraction was planned and in order to maximize the amount of available bone for implant placement, socket augmentation procedure was decided where a variant from conventional Bio-Col technique was suggested. As radiograph have revealed extensive bone loss pertaining to the distal aspect a two stage/ late implant was decided.

The procedure was explained to the patient and the consent was obtained for the same. Routine blood investigations were done. Prior to surgery PRF preparation was done [9]. Once PRF preparation is completed the patient is advised to rinse for 60 seconds with a $0.12 \%$ chlorhexidine mouth wash. After local anesthesia

Figure 1. Radiographic and clinical picture of splinted upper left central incisor.

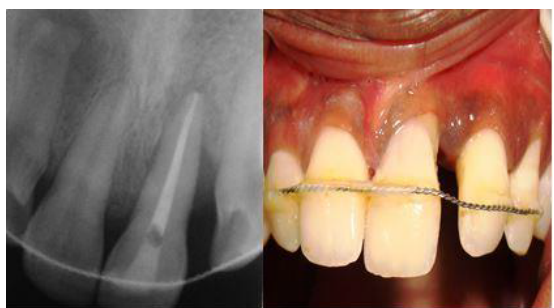

Figure 2. Atraumatic Extraction of upper left central incisor.

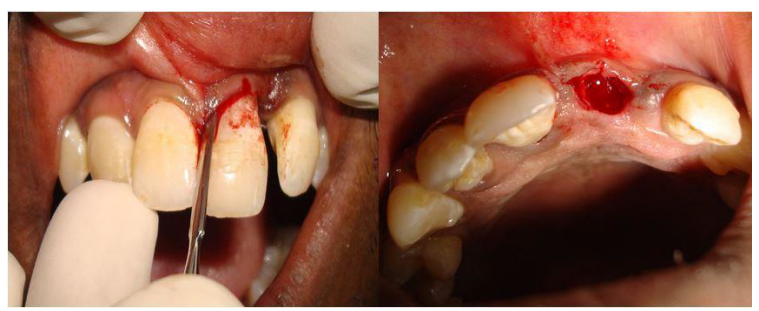


was achieved, sulcular incision with a $15 \mathrm{C}$ surgical blade was made circumferentially around upper left central incisor extending apically to the osseous crest. Next the tooth was extracted atraumatically utilizing a sharp Busar periosteal elevator and periotome (Figure 2) followed by the removal of granulation tissue and inspection of the socket.

A Lucas surgical curette is used to perform thorough curettage of the socket to remove any granulation tissue. Site was inspected for socket wall defects and for evidence of spontaneous bleeding. As spontaneous bleeding was not present, using small carbide bur socket walls were perforated for fresh bleeding. Bio-Oss bone graft was mixed with PRF over which the collagen dressing material [collaplug] was dipped in so that is uniformly soaked (Figure 3).Then the biomaterials were packed into the socket (Figure 4) with the finger pressure given over the facial gingiva to provide a "solid stop" to condense the graft against. Socket is closed with horizontal mattress sutures. Interim acrylic RPD with ovate pontic design was inserted. Immediate post-op IOPA radiograph was made pertaining to upper left central incisor [Figure 5]. Post-surgery instructions and removable partial denture instructions were provided. After 4 weeks, clinical examination showed no significant edema or infection and a satisfactory healing was noted.

A thorough follow up of 2 and 4 months revealed increased quantity of bone and good soft tissue contour (Figure 6A, 6B) and hence the implant placement was planned. After evaluating the clinical and radiographic findings, a tapered - self thread implant of $13 \mathrm{~mm}$ length and $4.5 \mathrm{~mm}$ diameter was considered ideal. Scaling and polishing was executed 2 weeks preceding to surgery. The prefabricated surgical stent was prepared to direct the implant placement in correct angulation. After anesthetizing the operating site via infiltration anesthesia, a full thickness (mucoperiosteal flap) was raised placing a horizontal paracrestal incision on the palatal part of the alveolar ridge. The flap was reflected to the labial surface to uncover the underlying bone. Opening on the occlusal area of the prefabricated surgical stent permitted the surgical burs to be positioned and angled correctly in the implant receiver site. The point of insertion on the bone was marked with the aid of a round bur introduced through the occlusal opening of the stent. This was followed by a the use of a $2 \mathrm{~mm}$ spiral drill at a bur speed of $800 \mathrm{rpm}$ to $1000 \mathrm{rpm}$ with profuse irrigation with normal saline. The depth stop of all instruments was placed at $13 \mathrm{~mm}$ corresponding to the designated implant length. The $2 \mathrm{~mm}$ spiral drill was taken to the predetermined depth followed by a $3 \mathrm{~mm}$ spiral drill which enlarged the opening along the angulations determined by the previous spiral drill. The recipient site was prepared to the final diameter $(4.5 \mathrm{~mm})$ by using manual osteotome of decided diameter by gentle tapping with the surgical mallet to successive increase in diameters. (Figure 7)

The implant was removed from the sterile packaging and placed in the prepared osteotomy site using implant carrier and manually screwed using torque wrench until the implant fitted snugly and could be rotated into place. After implant insertion, the covering screw was eased into position (Figure 8). The flap was closed over the implant and sutured using interrupted sutures. A temporary restoration was fabricated and used during the transitional period i.e. period of healing. After a healing period of 4 months, a minimal horizontal crestal incision just enough to expose the cover screw was made. The titanium covering screw was detached and swapped with a gingiva former which would allow the gingival margin to form properly during the healing period and guarantee an ideal emergence profile around the future crown abutment. The gingiva former after 2 weeks was interchanged with a transfer coping and impression was made with syringe material around the transfer coping and a heavy body material was positioned in the tray for the rest of the dentition. The gingiva former was negotiated back into the implant till the seating of the fabricated crown was to be done. The implant analog was united with the implant coping and secured with fastening screw. The abutment screws were threaded into position in the implant after removing the gingiva former and the final restoration was cemented to the abutment (Figure 9) The patient was kept on systematic maintenance appointments during which the esthetics \& functioning of the restoration were found acceptable. A one-year postoperative radiograph showed maintenance of adequate bone level and density around the implant (Figure 10).
Figure 3. Mixture of PRF, Bone graft, Colla plug.

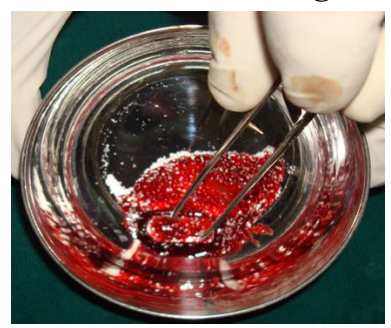

Figure 5. Immediate Post -operative radiograph of upper left central incisor tooth region.

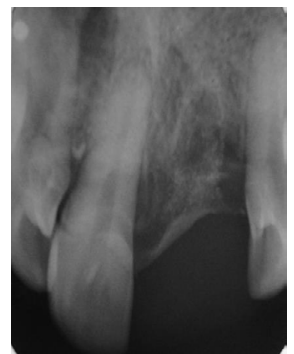

Figure 4. Biomaterials condensed into the socket.

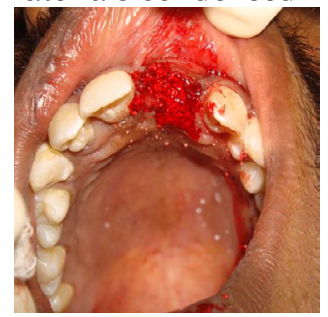

Figure 6A 6B. Four months post-operative radiograph, Clinical Picture.

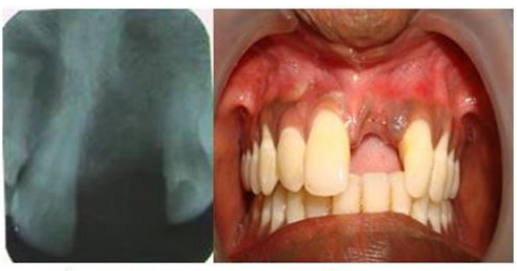

Figure 6A

Figure 6B 
Figure 7. Prepared implant site.

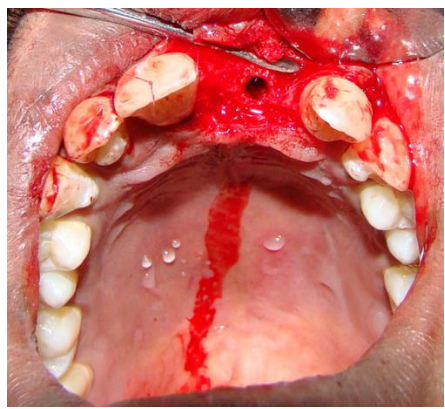

Figure 9. Final restoration cemented to abutment.

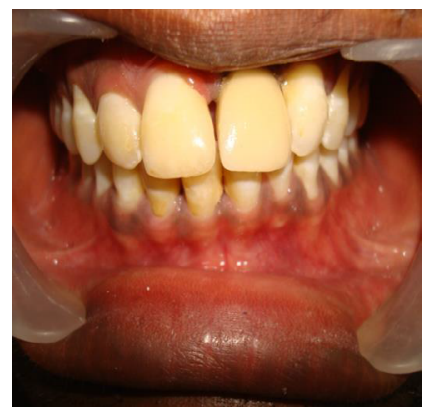

Figure 8. Implant placed in relation to upper left central incisor.

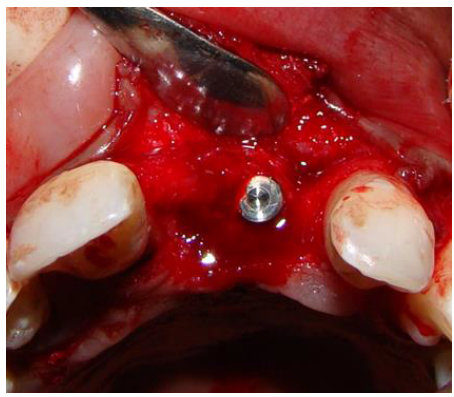

Figure 10. One-year Postoperative Radiograph.

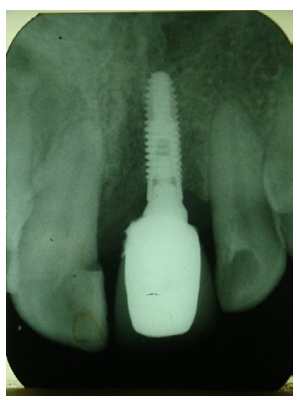

\section{Discussion}

The method offered in this article is a variant of the Bio-Col Technique first published by Sclar (1999) which preserves as much as possible the patient's existing alveolar bone and gingival contours during extraction [1]. Collagen dressing material serve as a matrix for soft tissue healing at the site. Usage of collagen wound dressing material was recommended not only to protect the graft material as majority of it may be lost due to configuration of the extraction socket but also to induce blood clot formation and stabilize the wound [10,11]. High biocompatibility and hemostatic ability that can enhance platelet aggregation thereby facilitating clot formation and wound stabilization makes collagen dressing most preferable under these conditions [12]. Moreover high chemotactic function of collagen for fibroblasts will promote cell migration and accelerate primary wound coverage [13]. PRF concentrates have been extensively used as an addition to tissue regeneration procedures $[14,15]$. The histologic analysis done on various studies showed a bone growth and a better trabecular organization in areas treated with PRF and FDBA, compared to control areas which where only treated with FDBA thereby decreasing the healing time so much that implant could be already placed 4 months after surgery [16]. The present case report demonstrated good radiographic bone fill, better soft tissue contours with faster healing time enabling the site to receive implant in a shorter time span supporting the literature.

Periodontally compromised states which mandates therapeutic extraction are one among the important clinical situations in which delayed implant placement is indicated. Clinician should recognize that considerable esthetic risk is associated with immediate replacement under these conditions, each of which can compromise the esthetic outcome secondary to soft recession and early or late osseous remodeling. Bone resorption rate will be more during the first 6 months following tooth extraction, unless a socket augmentation procedure is performed or implant is placed [17].
In general delayed implant placement is indicated when primary implant stability is not feasible due to acute infection, periodontal infection like abscess, defects of socket wall, bone defects like dehiscence and fenestration in the esthetic areas which can compromise the integrity of the buccal ridge crest. It is advisable to reevaluate the site augmented by Bio-Col technique after three months so that any site-development procedures if needed can be noted after inspecting the alveolar ridge contours and any residual esthetic defect that may have developed. Osseous defects at sites can be classified as favorable or unfavorable based on their morphology [5].

Regenerative potential of favorable defects are more as there is sufficient interdental bone remaining at these site to provide vascularity and cellularity to provide repair across the gap whereas in defects with unfavorable morphology interdental bone volume is less as the width of the wall defect is more than one third regenerative potential to provide complete osseous repair is difficult even when biomaterials such as grafts and barrier membranes are used. In esthetic sites managed with Modified Bio-Col technique if defects are favorable 4-6 months healing time is given prior to implant placement whereas if defects are unfavorable the Bio-Col technique serve as a space maintainer preventing the soft tissue collapse and scarring of the site making it suitable for site development procedures prior to implant placement.

Atraumatic preparation of the implant site determines quantity of vital bone surrounding the implant [18]. The threshold temperature level for bone survival is 47 degree $\mathrm{C}$ for 1 minute which when exceeded can result in osteolysis; this may contribute to a delay in osseointegration which explains detrimental effects of motorized drills used during osteotomy preparation of implant site [18-20]. Osteotomes are surgical instruments that can be used effectively to enhance the placement of dental implants [21, 22]. These instruments generally cut, compress or deform bone to assist in placement of implants. Deforming bone to accommodate the placement of an implant takes advantage of a unique 
molecular property of bone collagen by breaking the polymeric chains termed as sacrificial bonds between the collagen molecules. If the bone deformation associated implant placement can be accomplished without compromising blood supply healing of bone will not be jeopardized [23]. This study have utilized the same principle of using a combination of drill sequence along with osteotome usage to minimize bone resorption and maximize healing. Another reason is that these instruments gives the surgeon a tactile sense of the bone quality when compacting the bone , resulting in greater bone density, which is beneficial for initial implant support. In contrast to rotary instruments they serve to extend the apical floor of the osteotomy and have added advantage of not generating heat during use [24]. Hence preparation of an implant bed through the protocol followed in the current study through combination of initial rotary instruments followed by expansion- condensing osteotomes guarantees the maximum preservation of the remaining bone, improvement of primary implant stability, increased tactile sensitivity and major visibility and control of the job.

\section{Conclusion}

Accomplishment of a fruitful esthetic outcome and good patient satisfaction is a very challenging procedure, particularly when dealing with the esthetic area. Placement of a dental implant in the anterior esthetic zone is a technique-sensitive procedure with little room for error. The described variant of the Bio-Col technique in the study is a straightforward and reproducible procedure that has a biologic basis for success to potentiate sites for delayed implant placement. This standardized protocol allows successful and predictable long-term successful functional outcomes regarding alveolar bone regeneration and implant rehabilitation.

\section{References}

[1]. Sclar A. Ridge preservation for optimum esthetics and function. The Bio-Col technique. Postgrad Dent. 1999;6(1):3-11.

[2]. Lekovic V, Camargo PM, Klokkevold PR, Weinlaender M, Kenney EB, Dimitrijevic B, Nedic M. Preservation of alveolar bone in extraction sockets using bioabsorbable membranes. Journal of periodontology. 1998 Sep;69(9):1044-9.Pubmed PMID:9776033.

[3]. Aimetti M, Romano F, Griga FB, Godio L. Clinical and histologic healing of human extraction sockets filled with calcium sulfate. International Journal of Oral \& Maxillofacial Implants. 2009 Oct 1;24(5). PubmedPMID:19865631.

[4]. Van der Weijden F, Dell'Acqua F, Slot DE. Alveolar bone dimensional changes of post-extraction sockets in humans: a systematic review. Journal of clinical periodontology. 2009 Dec;36(12):1048-58.

[5]. Sclar AG. The Bio-Col Technique. In: Soft Tissue and Esthetic Considerations in Implant Therapy. Carol Stream, IL: Quintessence Publishing Co., 2003:75-112.

[6]. Norton MR, Wilson J. Dental implants placed in extraction sites implanted with bioactive glass: human histology and clinical outcome. International Journal of Oral \& Maxillofacial Implants. 2002 Mar 1;17(2).

[7]. Tonetti, MS, Hammerle, CH. European Workshop on Periodontology Group C. Advances in bone augmentation to enable dental implant placement: Consensus Report of the Sixth European Workshop on Periodontology. Journal of Clinical periodontology., 2008; 35(Suppl): 168-172. PubmedPMID:18724849.

[8]. Chen, ST,Buser, D. Clinical and esthetic outcomes of implants placed in postextraction sites. The International Journal of Oral \& Maxillofacial Implants., 2009 ;24(Suppl): 186-217. PubmedPMID:19885446.

[9]. Dohan DM, Choukroun J, Diss A, DohanSL, Dohan AJ, Mouhyi J, et al.Platelet-rich fibrin (PRF): a second-generation platelet concentrate. Part I: technological concepts and evolution. Oral Surg Oral Med Oral Pathol Oral RadiolEndod., 2006; 101:e37-44. PubmedPMID:16504849.

[10]. Wang HL, Kiyonobu K, Neiva RF. Socket augmentation: rationale and technique. Implant Dentistry. 2004 Dec 1;13(4):286-96.

[11]. Gupta D, Gundannavar G, Chinni DD, Alampalli RV. Ridge Preservation done ImmediatelyfollowingExtraction using Bovine Bone Graft, Collagen Plugand Collagen Membrane. International Journal of Oral Implantology and Clinical Research. 2012 Jan 1;3(1):8-16.

[12]. Sabelman EE. Biology, biotechnology and biocompatibility of collagen. Biocompatibility of tissue analogs. 1985:21-66.

[13]. Postlethwaite AE, Seyer JM, Kang AH. Chemotactic attraction of human fibroblasts to type I, II, and III collagens and collagen-derived peptides. Proceedings of the National Academy of Sciences. 1978 Feb 1;75(2):871-5.

[14]. Sakka S, Coulthard P. Bone quality: a reality for the process of osseointegration. Implant dentistry. 2009 Dec 1;18(6):480-5. Pubmed PMID: 20009601.

[15]. Dohan DM, Choukroun J, Diss A, Dohan SL, Dohan AJ, Mouhyi J, et al. Platelet-rich fibrin (PRF): a second-generation platelet concentrate. Part II: platelet-related biologic features. Oral Surgery, Oral Medicine, Oral Pathology, Oral Radiology, and Endodontology. 2006 Mar 1;101(3):e45-50.

[16]. Inchingolo F, Tatullo M, Marrelli M, Inchingolo AM, Scacco S, Inchingolo $\mathrm{AD}$, et al. Trial with Platelet-Rich Fibrin and Bio-Oss used as grafting materials in the treatment of the severe maxillar bone atrophy: clinical and radiological evaluations. Eur Rev Med Pharmacol Sci. 2010 Dec 1;14(12):107584.

[17]. Schwartz-Arad D, Chaushu G. The ways and wherefores of immediate placement of implants into fresh extraction sites: a literature review. Journal of periodontology. 1997 Oct;68(10):915-23.

[18]. Iyer S, Weiss C, Mehta A. Effects of drill speed on heat production and the rate and quality of bone formation in dental implant osteotomies. Part I: Relationship between drill speed and heat production. Int J Prosthodont. 1997; 10:411-414. Pubmed PMID:9495159.

[19]. Iyer S, Weiss C, Mehta A. Effects of drill speed on heat production and the rate and quality of bone formation in dental implant osteotomies. Part II: Relationship between drill speed and healing. Int J Prosthodont., 1997; 10:536-540. Pubmed PMID:9495174.

[20]. Tehemar S. Factors affecting heat generation during implant site preparation: a review of biologic observations and future considerations. Int J Oral MaxillofacImplants., 1999; 14:127-136. Pubmed PMID:10074763.

[21]. Garg AK. The use of osteotomes: a viable alternative to traditional drilling. Dent Implantol Update. 2002; 13:33-40. Pubmed PMID:12060956.

[22]. Saadoun AP, Le Gall MG.Implant site preparation with osteotomes: principles and clinical application. Pract Periodontics Aesthet Dent. Jun-Jul 1996;8(5):453-63.Pubmed PMID:9028267.

[23]. Yin XM, Dai JX, Wang XH, Xu DC, Zhong SZ. Observation of blood supplies system to mandible in transparent specimen. Shanghai kouqiangyixue= Shanghai journal of stomatology. 2003 Aug 1;12(4):266-8.

[24]. Hahn J. Clinical uses of osteotomes. Journal of Oral Implantology. 1999 Jan;25(1):23-9. 